\title{
Intra-aortic balloon pump treatment for patients with acute anterior wall myocardial infarction after reperfusion therapy
}

\author{
C.G. Feng ${ }^{1,2}$, X.J. Yang ${ }^{1}$, Q. Fu ${ }^{2}$, Y.J. Wang' ${ }^{2}$ H.Y. Ruan², Y. Lu ${ }^{2}$, \\ X.L. Li $^{2}$ and X.P. Zhang ${ }^{2}$ \\ ${ }^{1}$ Department of Cardiology, First Affiliated Hospital, Suzhou University, \\ Suzhou, China \\ ${ }^{2}$ Institute of Cardiovascular Disease of Xuzhou, Department of Cardiology, \\ Xuzhou Central Hospital, Xuzhou, \\ Jiangsu Province, China \\ Corresponding author: X.J. Yang \\ E-mail: xiangjunyangcn@126.com
}

Genet. Mol. Res. 13 (2): 4280-4288 (2014)

Received May 23, 2013

Accepted September 18, 2013

Published June 9, 2014

DOI http://dx.doi.org/10.4238/2014.June.9.14

\begin{abstract}
We evaluated the effects of an intra-aortic balloon pump on hemodynamics, brain natriuretic peptide concentration and cardiac function of patients with acute myocardial infarction, after reperfusion therapy. Sixty-three patients with acute anterior wall ST-elevation myocardial infarction who underwent primary percutaneous coronary intervention were given an intra-aortic balloon pump (32 cases) or not (control group, 31 cases). The mean pulmonary arterial pressure, pulmonary capillary wedge pressure and cardiac index were measured with a Swan-Ganz catheter. The brain natriuretic peptide concentration was detected by immunochemiluminometric assay. Left ventricular end-diastolic diameter and left ventricular ejection fraction were measured by echocardiography. No difference in baseline was observed between the two groups on day 1 in the hospital. On day 5, mean pulmonary artery pressure and pulmonary capillary wedge pressure of
\end{abstract}


patients with the intra-aortic balloon pump were significantly lower, and cardiac index of was higher than that of the controls, whereas no differences in left ventricular end-diastolic diameter or left ventricular ejection fraction were observed between the two groups. On days 5 and 90, the brain natriuretic peptide concentration of the intra-aortic balloon pump patients was lower than that of the controls. On day 90, left ventricular end-diastolic diameter was smaller in the intra-aortic balloon pump patients, but no difference in left ventricular ejection fraction was observed between the two groups. The intra-aortic balloon pump improved the hemodynamic index and cardiac function and decreased brain natriuretic peptide concentration in patients with acute anterior wall ST-elevation myocardial infarction.

Key words: Swan-ganz catheter; Brain natriuretic peptide; Acute myocardial infarction; Intra-aortic balloon pump;

Left ventricular end-diastolic diameter; Left ventricular ejection fraction

\section{INTRODUCTION}

Infarct-related artery occlusion for more than 30-40 min can cause myocardial necrosis (Jennings and Ganote, 1974; Jennings et al., 1975). Thus, numerous cases of necrotic and stunned myocardium are still discovered (Braunwald and Kloner, 1982) in patients with acute myocardial infarction (AMI) after successful reperfusion therapy [including intravenous thrombolysis, primary percutaneous coronary intervention (pPCI) and emergency coronary artery bypass graft (CABG) surgery]. Subsequently, complications of left ventricular remodelling and cardiac dysfunction occur. Generally, treatment after pPCI is based only on pharmacotherapy.

Intra-aortic balloon counterpulsation pumps (IABPs) are currently the most commonly used mechanical assist device for cardiac circulation. IABPs have significant efficacy on AMI complicated with cardiac shock or other high-risk situations (Buerke et al., 2012; Perera et al., 2013). Despite increased experience in frequency of and indications for using IABP, little is known about IABP applications in patients with AMI [ST-elevation myocardial infarction (STEMI)] after reperfusion therapy except for pharmacological treatment. Accordingly, this study was conducted to evaluate the effects of IABP on hemodynamics, brain natriuretic peptide (BNP) concentration and cardiac function in these patients.

\section{MATERIAL AND METHODS}

\section{General data}

A total of 63 patients in Xuzhou Central Hospital between June 2009 and June 2012 were selected as subjects for study. These patients underwent pPCI at the first onset of acute anterior wall STEMI and underwent successful reperfusion therapy as indicated by a quick reduction in the infarction associated ST segment after treatment. The patients 
included 38 males and 25 females aged 35-85 years whose target vessels were recanalization more than $3 \mathrm{~h}$ from onset. The inclusion criteria were as follows: 1) chest pain was more than $30 \mathrm{~min}$ and could not be relieved with nitroglycerin, 2) ST elevation $\geq$ $0.1 \mathrm{mV}$ in at least two limb leads or adjacent chest leads on the electrocardiogram (ECG; acute extensive anterior wall MI), and 3) troponin was normal or higher than normal upon hospital admission. The exclusion criteria were as follows: 1) a history of chronic myocardial infarction; 2) conditions affected the ST segment on ECG, such as left bundle branch block, idioventricular rhythm and application of cardiac pacemaker, etc.; and 3) unsatisfactory cardiac ultrasound image. Based on the optimum pharmaceutical therapy, the subjects were categorized into groups A (IABP group, 32 cases) and B (control group, 31 cases) according to IABP application. This study was conducted in accordance with the Declaration of Helsinki. This study was conducted with approval from the Ethics Committee of the Xuzhou Central Hospital, Xuzhou, China. Written informed consent was obtained from all participants.

\section{Therapeutic methods}

pPCI was performed on each patient. Postoperative coronary angiography indicated grade three of both thrombolysis in myocardial infarction (TIMI) and TIMI myocardial perfusion (TMP) according to the routine grading criterion for TIMI and TMP (The TIMI Study Group, 1985; Gibson et al., 2000). All patients without contraindication received the optimum pharmaceutical therapy after admission, including antiplatelet drugs, anticoagulants, statins, angiotensin converting enzyme inhibitors or angiotensin II receptor antagonists, beta receptor blockers, nitrate medicine and drugs for blood pressure control and glycemic control, etc.

\section{ECG}

The patients were subjected to ECG as soon as they were admitted to the hospital and $1 \mathrm{~h}$ after pPCI. The sum of ST elevation $\left(\sum \mathrm{ST}\right)$ was calculated with the depression magnitude of the ST segment by the formula of [ $\sum \mathrm{ST}$ (admission) - $\sum \mathrm{ST}$ (after $\mathrm{pPCI})] / \sum \mathrm{ST}$ (admission) (Feldman et al., 2003). The subjects were categorized into two groups according to the depression magnitude of ST segment: rapid depression group (depression $\geq 50 \%$ ) and continuous elevation group (depression $<50 \%$ ). Rapid ST depression was considered as high performance for reperfusion (Tomaszuk-Kazberuk et al., 2002, 2004).

\section{IABP implantation and mode}

Under sterile operation, the patients were implanted with a balloon catheter after femoral artery puncture by the Seldinger technique. 8-French Datascope ${ }^{\circledR}$ catheters and a Datascope $^{\circledR} 2000$ IABP system (Datascope Corporation, Fairfield, NJ, USA) were used in all studied patients.

The size of balloon counterpulsation was determined by the height of each patient, i.e., 30-, 40-, and 50-mL balloons for 152-163, 163-183, and $>183 \mathrm{~cm}$-tall patients, respec- 
tively. Under fluoroscopy, the balloon was oriented towards the descending aorta $1-2 \mathrm{~cm}$ below the left subclavian artery and connected to the aortic balloon counterpulsation machine from Datascope under the ECG trigger mode. Counterpulsation was performed at a ratio of $1: 1$. About $2 \mu \mathrm{L}$ heparin were injected into the central cavity of the balloon catheter every 30 min to prevent thrombosis on the balloon surface, by which the ACT was maintained between 200 and $300 \mathrm{~s}$. IABP was applied for over 5 days.

\section{Implantation of Swan-Ganz catheter}

The implantation of a Swan-Ganz catheter (Edward Lifesciences, LLC, Irvine, CA, USA) was performed after pPCI beside the bed where the pressure waveform of each cardiac chamber was visualized by the monitor. A catheter was implanted by internal jugular venipuncture, which passed through the precava into the right atrium, right ventricle and pulmonary artery. When the balloon was inflated, the catheter went into the distal pulmonary artery and then went back to the original position in the pulmonary artery resulting from balloon deflation, which indicated that the catheter was in a good position. Afterwards, the catheter was anchored by stitching on the skin, and the puncture site was covered by a sterile dressing before tape fixation.

\section{BNP test}

The cubit venous blood of patients in groups A and B was drawn 1 day after admission (before pPCI), 5 days after admission (after IABP), and 90 days after admission to measure the BNP concentration using a Triage BNP detector (Biosite Company, San Diego, CA, USA). The normal value was $0-120 \mathrm{pg} / \mathrm{mL}$.

\section{Ultrasonic cardiogram (UCG) examination}

UCG examination was performed in the two groups on days 1 , 5, and 90 after admission. A Philips Sonos 5500 echocardiographic device and X4 detector (Philips Medical Systems, Andover, MA, USA) were used at a frequency of 2-4 MHz. The standard for chamber view of the cardiac apex was selected and the average LVEF was assessed by a modified Simpson method from three cardiac cycles. The long-axis-section view of papillary muscles beside the sternum was selected, and the average LVEDD was determined by M-mode echocardiography from three cardiac cycles.

\section{Statistical analysis}

Statistical analysis was performed using the SPSS 17.0 software, and the measurement data were presented as means $\pm \mathrm{SD}$. The $t$-test of two independent samples and chi-square test with a fourfold table (value corrected was used when the theoretical value was $<5$ ) were used to compare the measurement and enumeration data between groups A and B and for the enumeration data by the chi-square test. $\mathrm{P}<0.05$ was considered as a significant difference.

\section{RESULTS}




\section{General results}

No significant differences in general data, time from onset until target blood vessel dredging, basic indices of hemodynamics and cardiac function were observed between groups A and B upon hospital admission. The data are summarized in Table 1.

\begin{tabular}{|c|c|c|c|c|}
\hline & Group A $(\mathrm{N}=32)$ & Group B $(\mathrm{N}=31)$ & $\mathrm{T} / \chi^{2}$ & $\mathrm{P}$ \\
\hline Age (years old) & $64.41 \pm 11.85$ & $61.97 \pm 13.29$ & 0.769 & 0.445 \\
\hline Male & $22(22 / 32)$ & $19(19 / 31)$ & 0.39 & 0.54 \\
\hline Hypertension & 12 & 17 & 1.91 & 0.71 \\
\hline Dyslipidemia & 14 & 9 & 1.47 & 0.23 \\
\hline Type 2 diabetes & 13 & 10 & 0.48 & 0.49 \\
\hline Smoking history (within one year) & 14 & 9 & 1.47 & 0.23 \\
\hline Time from onset until target vessel was dredged (h) & $6.13 \pm 1.64$ & $5.74 \pm 1.80$ & 0.598 & 0.893 \\
\hline MPAP $(\mathrm{mmHg})$ & $27.00 \pm 4.64$ & $25.81 \pm 4.07$ & 0.456 & 1.084 \\
\hline PCWP (mmHg) & $22.66 \pm 4.17$ & $21.65 \pm 4.05$ & 0.574 & 0.976 \\
\hline $\mathrm{CI}\left[\mathrm{L} /\left(\mathrm{min} \cdot \mathrm{m}^{2}\right)\right]$ & $2.61 \pm 0.37$ & $2.69 \pm 0.39$ & 0.836 & 0.859 \\
\hline $\mathrm{BNP}(\mathrm{pg} / \mathrm{mL})$ & $232.69 \pm 71.16$ & $218.03 \pm 66.85$ & 0.629 & 0.842 \\
\hline LVEDD (mm) & $51.03 \pm 3.35$ & $49.19 \pm 3.36$ & 0.779 & 2.172 \\
\hline $\operatorname{LVEF}(\%)$ & $43.13 \pm 3.48$ & $42.97 \pm 3.59$ & 0.555 & 0.710 \\
\hline
\end{tabular}

$\mathrm{MPAP}=$ mean pulmonary artery pressure $\mathrm{PCWP}=$ pulmonary capillary wedge pressure $\mathrm{CI}=$ cardiac index $; \mathrm{BNP}=$ brain natriuretic peptide; $\mathrm{LVEDD}=$ left ventricular end-diastolic diameter; $\mathrm{LVEF}=$ left ventricular ejection fraction.

\section{Indications and results of IABP using}

According to the optimum pharmaceutical therapy, the application of IABP resulted in changes in BNP, LVEDD, and LVEF, as shown in Table 2.

Along with the pharmaceutical treatment and application of IABP, the symptoms and other indices in patients improved. No death or target vessel revascularization was recorded until the last of the 90-day follow-up. Three and seven cases in groups A and B were re-admitted to the hospital because of acute cardiac insufficiency. On day 5, the hemodynamics indices in group A were superior to those in group B, i.e., the mean pulmonary artery pressure (mPAP) and pulmonary capillary wedge pressure (PCWP) distinctly decreased, and the cardiac index (CI) clearly increased. On days 5 and 90, the serum BNP concentration in the IABP group was lower than that in the control group, whereas LVEDD was smaller than that in the IABP group on day 90 .

\section{Complications of IABP}

Nine cases of local hematoma $(9 / 32,28.1 \%)$, one case of infection at puncture site $(1 / 32$, $3.1 \%)$, three cases of lower limb ischemia $(3 / 32,9.4 \%)$ and two cases of thrombocytopenia $(2 / 32,6.3 \%)$ were found, and all of them recovered after treatment. Balloon burst occurred in one patient without sequel. None of the complications above were observed in the control group. The patient with hematoma at the puncture site did not show an apparent decrease in hemoglobin. The standard for plastocyte reduction was a count less than $100 \times 10^{9} / \mathrm{L}$, which was restored after stopping IABP. The patients with lower limb ischemia did not develop acra gangrene, etc. 


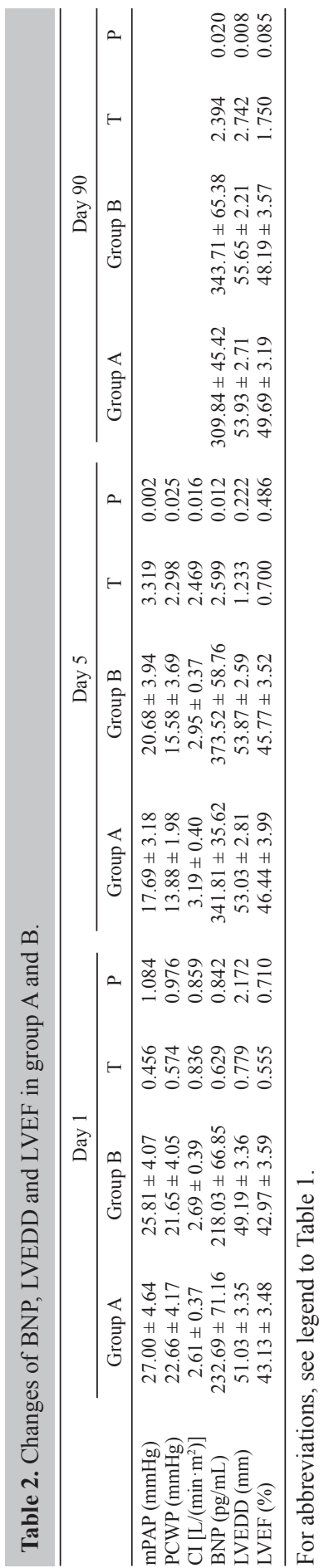




\section{DISCUSSION}

This study mainly found that using IABP in patients with acute anterior wall STEMI after reperfusion therapy improved the hemodynamic index and cardiac function and decreased the BNP concentration.

IABP is a mechanical assist device for cardiac circulation that functions as follows (Jones et al., 2012): 1) IABP balloon deflates in end diastole before aortic valve opening to reduce intra-aortic end-diastolic pressure and left ventricular afterload, and 2) balloon inflates in early diastole after aortic valve closing to elevate peak diastolic pressure and coronary perfusion pressure in stenotic and normal arteries as well as to support blood circulation of brain, kidney and surrounding organs. Some research has demonstrated that IABP increases the CI by $10-40 \%$ (Moreno et al., 2000) compared with simple drug therapy and decreases inflammation in patients (Prondzinsky et al., 2010). At present, IABP is mainly used to treat AMI complicated with cardiac shock (Buerke et al., 2012) and to intervene with critical disease as adjuvant therapy (Gu et al., 2011; Jones et al., 2012; Parissis et al., 2012; Perera et al., 2013).

Myocardial stunning or post-ischemic myocardial dysfunction is the mechanical dysfunction that persists after reperfusion despite restoration of normal coronary flow in infarction-related arteries (Braunwald and Kloner, 1982). It is actually a delayed recovery process of sublethal myocardium with reversible damages from ischemia after reperfusion, in which myocardial necrosis has not yet occurred but the structure and metabolism changes. In particular, systolic dysfunction is restored after several hours, days or weeks after reperfusion (Kloner et al., 1998). Infarct-related artery occlusion for more than 30-40 min can cause myocardial necrosis (Jennings et al., 1975). Thus, numerous cases of necrotic and stunned myocardium are still discovered (Braunwald and Kloner, 1982) in patients with AMI after successful reperfusion therapy (including intravenous thrombolysis, pPCI and emergency CABG surgery). Subsequently, complications of left ventricular remodelling and cardiac dysfunction occur. On the other hand, anomalous expansion of ventricular myocardium during systole further reduces left ventricular output. When the necrotic muscle cells mutually move, the infarction region is stretched to become thinner and longer, especially in patients with extensive anterior wall MI. Consequently, local tension increases and the entire myocardial wall expands according to Laplace's law from the first few hours to several days. Ventricular dilatation initially occurs in other patients, which leads to a vicious circle of dilatation (Pfeffer and Braunwald, 1990; Braunwald and Pfeffer, 1991). Therefore, the key to stopping this vicious circle is successful reperfusion and perioperative treatment.

Since the Swan-Ganz catheter was created in 1970, it has been extensively used to monitor hemodynamics in critical patients. This catheter has also been considered as the gold standard for cardiac failure and a symbol of critical care medicine (Dalen, 2001). BNP is a 32-amino acid polypeptide hormone secreted by ventricular myocytes with a half-life of $20 \mathrm{~min}$. BNP secretion and release increase in response to reduced left ventricular systolic function and enhanced atrial and ventricular transmural pressure during diastole, which is an important indicator for diagnosis, efficacy and prognosis of cardiac insufficiency (Seo et al., 2011; Reesukumal and Pratumvinit, 2012).

Our research focused on patients with acute anterior wall STEMI whose target vessels were dredged within $3 \mathrm{~h}$ from the onset. The partial myocardial necrosis and myocardium stunning caused the hemodynamic parameters (mPAP, PCWP, CI) and BNP level to be signifi- 
cantly lower than the normal level upon hospital admission; even the reperfusion therapies for the patients were successful. With the optimum pharmaceutical treatment and IABP therapy, the above parameters were superior to those of the control group 5 days after performing IABP, and these effects continued after stopping IABP. The BNP level of patients in the implantation group was still lower than that of the control group during the follow-up after 90 days in the hospital. The LVEDD of patients in the two groups showed no significant difference on day 5 , which was probably correlated with the short infarction time. On day 90, the LVEDD was lower than that of the control group, which indicated a more significant remodelling of left ventricle in the control group patients. The autoregulation and treatment with modern medicine led to LVEFs showing no remarkable difference between the two groups on days 5 and 90, but the LVEF showed an increasing trend in the implantation group. Therefore, patients with acute anterior wall STEMI should be treated with an IABP after pPCI based on the optimum medicine therapy to improve coronary artery perfusion, reduce the cardiac workload, increase the cardiac output and reduce the neuroendocrine activity of the body. Consequently, left ventricle remodelling is alleviated and cardiac function is improved, ultimately leading to a high success rate of rescue and improved long-term prognosis of patients. Consistent with another study (Lauten et al., 2012), side effects such as local hematoma and ischemia in distal limb, etc. increased in the IABP implantation group compared with the control group, but no serious complication was observed.

Many recent studies have concluded that IABP cannot reduce the risk of death when applied in acute STEMI patients with or without shock who had received pPCI (Mahmoudi et al., 2012; Sjauw et al., 2012; Thiele et al., 2012). However, our study showed that according to the indicators of hemodynamic, ultrasound echocardiography and BNP as endpoint, the results and clinical symptoms significantly changed with IABP. Mortality and other major adverse cardiac events (MACEs) need to be included in the future.

Our study has several disadvantages. First, statistical deviation was high because of the small sample pool, which also resulted in difficulty counting the MACEs. Second, several parameters such as the area of myocardial infarction were not included because of the limited conditions. Third, we selected patients only with anterior wall and/or extensive anterior wall MI whose target vessels were dredged more than $3 \mathrm{~h}$ from the onset. Further research on patients with shorter onset or smaller area of MI is required to determine whether IABP implantation is necessary and affects the parameters observed.

\section{ACKNOWLEDGMENTS}

Research supported by the "Jiangsu Medical Leading Talent" Project (\#LJ201140), and National Natural Science Foundation of 2010 (\#K112213810).

\section{REFERENCES}

Braunwald E and Kloner RA (1982). The stunned myocardium: prolonged, postischemic ventricular dysfunction. Circulation 66: 1146-1149.

Braunwald E and Pfeffer MA (1991). Ventricular enlargement and remodeling following acute myocardial infarction: mechanisms and management. Am. J. Cardiol. 68: 1D-6D.

Buerke M, Prondzinsky R, Lemm H, Dietz S, et al. (2012). Intra-aortic balloon counterpulsation in the treatment of infarction-related cardiogenic shock-review of the current evidence. Artif. Organs 36: 505-511. 
Dalen JE (2001). The pulmonary artery catheter-friend, foe, or accomplice? JAMA 286: 348-350.

Feldman LJ, Coste P, Furber A, Dupouy P, et al. (2003). Incomplete resolution of ST-segment elevation is a marker of transient microcirculatory dysfunction after stenting for acute myocardial infarction. Circulation 107: 2684-2689.

Gibson CM, Cannon CP, Murphy SA, Ryan KA, et al. (2000). Relationship of TIMI myocardial perfusion grade to mortality after administration of thrombolytic drugs. Circulation 101: 125-130.

Gu J, Hu W, Xiao H, Feng X, et al. (2011). Prophylactic intra-aortic balloon pump reduces C-reactive protein levels and early mortality in high-risk patients undergoing percutaneous coronary intervention. Acta Cardiol. 66: 499-504.

Jennings RB and Ganote CE (1974). Structural changes in myocardium during acute ischemia. Circ. Res. 35 (Suppl 3): 156-172.

Jennings RB, Ganote CE and Reimer KA (1975). Ischemic tissue injury. Am. J. Pathol. 81: 179-198.

Jones HA, Kalisetti DR, Gaba M, McCormick DJ, et al. (2012). Left ventricular assist for high-risk percutaneous coronary intervention. J. Invasive Cardiol. 24: 544-550.

Kloner RA, Bolli R, Marban E, Reinlib L, et al. (1998). Medical and cellular implications of stunning, hibernation, and preconditioning: an NHLBI workshop. Circulation 97: 1848-1867.

Lauten P, Rademacher W, Goebel B, Kretzschmar D, et al. (2012). Intra-aortic counterpulsation for hemodynamic support in patients with acute ischemic versus non-ischemic heart failure. J. Invasive Cardiol. 24: 583-588.

Mahmoudi M, Hauville C, Gaglia MA, Jr., Sardi G, et al. (2012). The impact of intra-aortic balloon counter-pulsation on in-hospital mortality in patients presenting with anterior ST-elevation myocardial infarction without cardiogenic shock. Cardiovasc. Revasc. Med. 13: 328-330.

Moreno R, Garcia E, Abeytua M, Soriano J, et al. (2000). Early coronary angioplasty for acute myocardial infarction complicated by cardiogenic shock: have novel therapies led to better results? J. Invasive Cardiol. 12: 597-604.

Parissis H, Soo A and Al-Alao B (2012). Intra-aortic balloon pump (IotaAlphaBetaRho): from the old trends and studies to the current "extended" indications of its use. J. Cardiothorac. Surg. 7: 128.

Perera D, Stables R, Clayton T, De SK, et al. (2013). Long-term mortality data from the balloon pump-assisted coronary intervention study (BCIS-1): a randomized, controlled trial of elective balloon counterpulsation during high-risk percutaneous coronary intervention. Circulation 127: 207-212.

Pfeffer MA and Braunwald E (1990). Ventricular remodeling after myocardial infarction. Experimental observations and clinical implications. Circulation 81: 1161-1172.

Prondzinsky R, Lemm H, Swyter M, Wegener N, et al. (2010). Intra-aortic balloon counterpulsation in patients with acute myocardial infarction complicated by cardiogenic shock: the prospective, randomized IABP SHOCK Trial for attenuation of multiorgan dysfunction syndrome. Crit. Care Med. 38: 152-160.

Reesukumal K and Pratumvinit B (2012). B-type natriuretic peptide not TIMI risk score predicts death after acute coronary syndrome. Clin. Lab. 58: 1017-1022.

Seo SM, Kim S, Chang K, Min J, et al. (2011). Plasma B-type natriuretic peptide level can predict myocardial tissue perfusion in patients undergoing primary percutaneous coronary intervention for acute ST-segment elevation myocardial infarction. Coron. Artery Dis. 22: 405-410.

Sjauw KD, Engström AE, Vis MM, Boom W, et al. (2012). Efficacy and timing of intra-aortic counterpulsation in patients with ST-elevation myocardial infarction complicated by cardiogenic shock. Neth. Heart J. 20: 402-409.

The TIMI Study Group (1985). The thrombolysis in myocardial infarction (TIMI) trial. N. Engl. J. Med. 312: 932-936.

Thiele H, Schuler G, Neumann FJ, Hausleiter J, et al. (2012). Intraaortic balloon counterpulsation in acute myocardial infarction complicated by cardiogenic shock: design and rationale of the Intraaortic Balloon Pump in Cardiogenic Shock II (IABP-SHOCK II) trial. Am. Heart J. 163: 938-945.

Tomaszuk-Kazberuk A, Korecki J, Kochman W, Dobrzycki S, et al. (2002). Rapid resolution of ST segment elevation predicts recovery of left myocardial contraction in patients with acute myocardial infarction treated with percutaneous coronary angioplasty. Przegl. Lek. 59: 638-641.

Tomaszuk-Kazberuk A, Musial WJ, Dobrzycki S and Korecki J (2004). Normalisation of elevated ST segment predicts return of left ventricular systolic function and improved outcome in patients with acute myocardial infarction, treated with primary coronary angioplasty. Kardiol. Pol. 60: 541-549. 